\title{
Diseño y validación de una escala de actitudes de los profesionales asistenciales hacia la liberación del uso de sujeciones en personas dependientes
}

\section{Development and validation of a scale of healthcare professional attitudes to the liberation from restraint use with dependent people}

\author{
M. Villacieros ${ }^{1}$, L. Steegman ${ }^{1}$, M.L. Mejías ${ }^{1}$, J.C. Bermejo ${ }^{2}$
}

\section{RESUMEN}

Fundamento. En nuestro entorno ya existen estudios sobre prevalencia y uso de sujeciones pero no un instrumento de medida que elimine la subjetividad del evaluador para su uso en clínica y en investigación. Objetivo: diseño y validación de una escala de medida de la actitud del profesional asistencial hacia el uso de las sujeciones físicas y farmacológicas.

Método. Estudio descriptivo y correlacional de validación de escala. Tras revisión bibliográfica, se elaboró un listado de ítems que se sometieron a análisis de validez; de contenido mediante jueces, de criterio mediante contraste con variable externa y de constructo mediante análisis factorial exploratorio. La fiabilidad mediante coeficiente alfa de Cronbach.

Resultados. Contestaron al cuestionario 276 personas, 224 mujeres $(82,4 \%)$ y 48 hombres $(17,6 \%)$, media de edad de 38,65 años. La escala con 40 ítems ofreció un alfa de Cronbach 0,90 . Se obtuvieron 4 dimensiones que explicaban un $46,76 \%$ de la varianza total. Se nombraron los factores Autonomía (15,08\% de la varianza), Seguridad (12,23\%), Entorno (8,46\%) y Evitación (6,06\%). Salvo la última, todas las subescalas obtuvieron un alfa superior a 0,72 . Se obtuvieron diferencias debido a la experiencia en el cuidado del Alzheimer. El personal con experiencia puntúa significativamente más bajo en Factor 1 y Factor 3 y más alto en Factor 2, pero sin diferencias en el cómputo global. También por género, las mujeres puntuaron en Factor 1 más alto.

Conclusión. Esta escala constituye un paso significativo en el estudio de las actitudes de los profesionales asistenciales profundizando en las causas subyacentes.

Palabras clave. Actitudes. Sujeciones. Geriatría. Escala.

\begin{abstract}
Background. There are studies on the prevalence and use of restraints but not a measuring instrument to eliminate subjectivity in evaluation of their use in clinical practice and research in our environment. Objective. Design and validation of a scale of measurement of attitudes of health care professionals towards the use of physical and pharmacological restraints.

Methods. Descriptive and correlational study for scale validation. Following a literature review, we developed a list of items that were subjected to a validity analysis: validity of content through judges, validity of criterion through external variable contrast, and validity of construct using exploratory factor analysis. Reliability was analysed using the Cronbach alpha coefficient.

Results. Two hundred and seventy-six people answered the questionnaire, $82.4 \%$ (224) women and $17.6 \%$ (48) men, average age 38.65 years. The 40 item scale provided a Cronbach alpha of 0.897 . Four dimensions were obtained explaining $46.758 \%$ of the total variance. The factors were named: Autonomy (15.084\% of the variance), safety (12.228\%), environment (8.463\%) and avoidance $(6.062 \%)$. Except for the last one, all the subscales obtained an alpha superior to 0.717 . There were differences due to the experience in Alzheimer care. Staff with experience score significantly lower in Factor 1 and Factor 3, and higher in Factor 2, but there is no difference in the overall computation. Moreover, by gender, women scored higher in Factor 1.
\end{abstract}

Conclusions. This scale is a significant step forward in the study of the attitudes of health care professionals as it enquires into underlying causes.

Kew words. Attitudes. Restraint. Geriatric. Scale.
1. Comité de Ética Asistencial San Camilo.

2. Centro de Humanización de la Salud.

Recepción: 7 de agosto de 2013

Aceptación provisional: 30 de septiembre de 2013

Aceptación definitiva: 5 de noviembre de 2013

\author{
Correspondencia: \\ Marta Villacieros \\ Centro de Humanización de la Salud \\ Dpto. Investigación \\ C/ Sector Escultores, 39 \\ 28760 Tres Cantos \\ Madrid \\ investigación@humanizar.es
}




\section{INTRODUCCIÓN}

La utilización de sujeciones para una mejor atención de las personas mayores o con discapacidad ha sido una práctica casi habitual, especialmente en el caso de personas con algún tipo de demencia, con la intención de proteger a la persona dependiente.

Entendemos por sujeciones todos aquellos mecanismos de contención, sean físicos o farmacológicos, aplicados a personas dependientes como medidas de seguridad, por ejemplo para evitar caídas, conductas disruptivas o complicaciones de otros dispositivos médicos ${ }^{1}$.

Desde el punto de vista ético, la restricción física o farmacológica constituye una vulneración del principio de autonomía. La dignidad de la persona se pone en peligro cada vez que se limita su capacidad de movimiento. Tal limitación de la libertad de la persona solo puede estar justificada en virtud del respeto al principio de beneficencia, solamente se justificaría la sujeción si se hiciera en mayor beneficio de la persona sobre la que se aplica.

Puesto que las sujeciones físicas se han asociado también a efectos adversos o daños derivados de su uso como lesiones, mortalidad, reducción del bienestar psicológico, detrimento de capacidades cognitivas y reducción en la movilidad ${ }^{2}$, existe un debate abierto sobre la conveniencia o no de su uso.

Así, en las últimas décadas se ha producido a nivel internacional un punto de inflexión favorable hacia el menor uso de las sujeciones por lo que su prevalencia ha disminuido sensiblemente en la mayoría de los países sobre los que se dispone de información. Alemania, Austria, Dinamarca o Países Bajos se encuentran en vanguardia. En España, aunque no existe legislación a nivel estatal, se manifiestan diversas leyes y decretos de los servicios sociales autonómicos en Cantabria, Cataluña, Navarra o Valencia y se plantea investigación específica en centros de cuidados prolongados con el Programa Desatar al Anciano y al Enfermo de Alzheimer ${ }^{3}$.

Cada vez son más los estudios que obtienen datos en contra del uso de suje- ciones: un estudio realizado en 25 residencias públicas de la Comunidad de Madrid concluye que probablemente exista un uso excesivo de barandillas que afecta incluso a residentes sin deterioro cognitivo ni dependencia física ${ }^{4}$. Existen también estudios y revisiones donde se recogen gran cantidad de efectos negativos sobre el dependiente como incontinencia, deterioro funcional agudo, úlceras de presión, comportamientos regresivos, incluso la muerte en periodos muy cortos de tiempo ${ }^{5-9}$.

En la misma línea proliferan los estudios cuyos resultados, después de la implementación de programas de liberación de sujeciones en residencias, muestran que entre otros beneficios se da una mejora del equilibrio, una importante reducción del número de caídas, la práctica desaparición de caídas con consecuencias graves, la mejora en el estado de salud con el consecuente aumento de satisfacción tanto de los usuarios como de sus familias y una sensible disminución del burnout del personal auxiliar ${ }^{10-12}$.

En España, el Proyecto Libera-Ger perteneciente a la Fundación Pilares, tiene como objetivo eliminar el uso de sujeciones en los centros de atención sociosanitaria, estableciendo los beneficios objetivos, físicos, psicoemocionales y sociales, de la no sujeción, sin abandonar la seguridad de la persona dependiente. Aun así, en nuestro país, la prevalencia de uso del $23 \%$ en los centros residenciales para personas mayores, es la cifra más alta de los países europeos ${ }^{13}$.

Entre las justificaciones que mantienen su uso como el riesgo de caídas y los problemas de conducta también hemos de considerar los casos que obedecen a una serie de razones no expresadas, como la autoprotección del profesional, de la familias, o del centro (progresivo aumento de la carga asistencial, déficit de motivación del personal, carencia de formación, ausencia de normas al respecto, tolerancia social...), incluso lo que en algunas instancias se califica de mitos ${ }^{14,3}$ extendidos entre los propios profesionales: las sujeciones corrigen la postura, las sujeciones previenen la interferencia con 
el tratamiento médico, la falta de personal idóneo hace necesario el uso de sujeciones, atender a las personas es más fácil y se realiza de forma más eficiente mediante el uso de sujeciones...

Algunos estudios realizados sobre factores que favorecen el mayor o menor uso de sujeciones físicas concluyen que uno de los discriminantes más potentes es la actitud del personal asistencial hacia las mismas $^{15,16}$. Por ejemplo, cuando se estudian las actitudes entre personal de enfermería se ve que las respuestas emocionales son variadas incluyendo tristeza y culpa, que coexisten con manifestaciones sobre la seguridad que aporta su uso ${ }^{17}$. Existe una ambivalencia emocional que genera conflictos, ya que la mayoría sienten pena por el anciano y tienen sentimientos negativos hacia el uso de las sujeciones a la vez que la liberación les genera dificultades.

En paralelo existen movimientos encaminados a sensibilizar y promover el desarrollo de la calidad de vida de las personas dependientes entre los diferentes agentes sociales que están en contacto con ellas (profesionales del cuidado, familiares y organismos públicos). Cada vez son más las iniciativas que pretenden formar al personal en torno a decisiones que están totalmente traspasadas por la dimensión de la ética profesional ${ }^{18}$. En el diálogo sobre cuestiones éticas se puede observar cómo el auditorio que participa modifica su opinión sobre la necesidad del uso de las mismas, mientras que la respuesta inicial contesta que depende del caso, en la respuesta final contesta que no, normalmente $^{19}$.

Dicho todo ello, parece razonable recomendar que el número de sujeciones utilizadas en los centros sea cada vez menor. Deben establecerse objetivos e indicadores que permitan un control máximo de las restricciones y de su progresiva disminución, avanzando hacia el escenario ideal en el que las sujeciones dejen de utilizarse. Pero para que se dé un cambio real en los centros socio sanitarios y residencias también es necesario un cambio cultural entre los profesionales dirigido al uso racional y adecuado de las sujeciones; es conve- niente asegurarse de que el criterio para establecer una sujeción no sea defensivo; por ejemplo, que el miedo a posibles responsabilidades penales de la institución o los profesionales no sea el que provoque la aplicación de la restricción.

Entendemos necesaria la creación de herramientas de trabajo para poder ampliar la investigación. Los instrumentos que contemplan un abanico amplio de posibilidades o respuestas como las escalas o cuestionarios, sin estar exentos de limitaciones, son necesarios principalmente para eliminar el factor subjetivo del evaluador y facilitar la investigación ${ }^{20}$.

Aunque en la literatura se encuentran herramientas de medida de actitud hacia intervenciones restrictivas ${ }^{21}$ como el Cuestionario de Actitudes Mastricht (Maastricht Attitude Questionnaire) que recoge la actitud de enfermería hacia las restricciones físicas $^{22,23}$, en nuestro país no existe una herramienta que mida la actitud del profesional asistencial hacia el uso de las sujeciones.

En este sentido, el objetivo de este estudio es el diseño y validación de una escala de medida de la actitud del profesional asistencial sociosanitario hacia el uso de las sujeciones, sean físicas o farmacológicas.

\section{MÉTODO}

Diseño del estudio: descriptivo y correlacional. Estudio piloto para la validación de una escala de medida de actitudes del personal asistencial de centros sociosanitarios hacia el uso de las sujeciones.

\section{Participantes}

De los 421 asistentes a las Jornadas de Alzheimer 2013, se recogieron 299 cuestionarios, de los cuales 23 estaban incompletos al menos en la mitad de los ítems, por lo que se descartaron. El resto, 276, constituyeron la muestra de nuestro trabajo. El $82,4 \%$ (224) eran mujeres y el 17,6\% (48) hombres, 4 personas no respondieron a esta pregunta. La media de edad resultó 38,65 años (DT: 13,28) (Tabla 1). 
Tabla 1. Descripción de la muestra en cuanto a variables sociodemográficas y vinculación con el cuidado del Alzheimer (formación y experiencia).

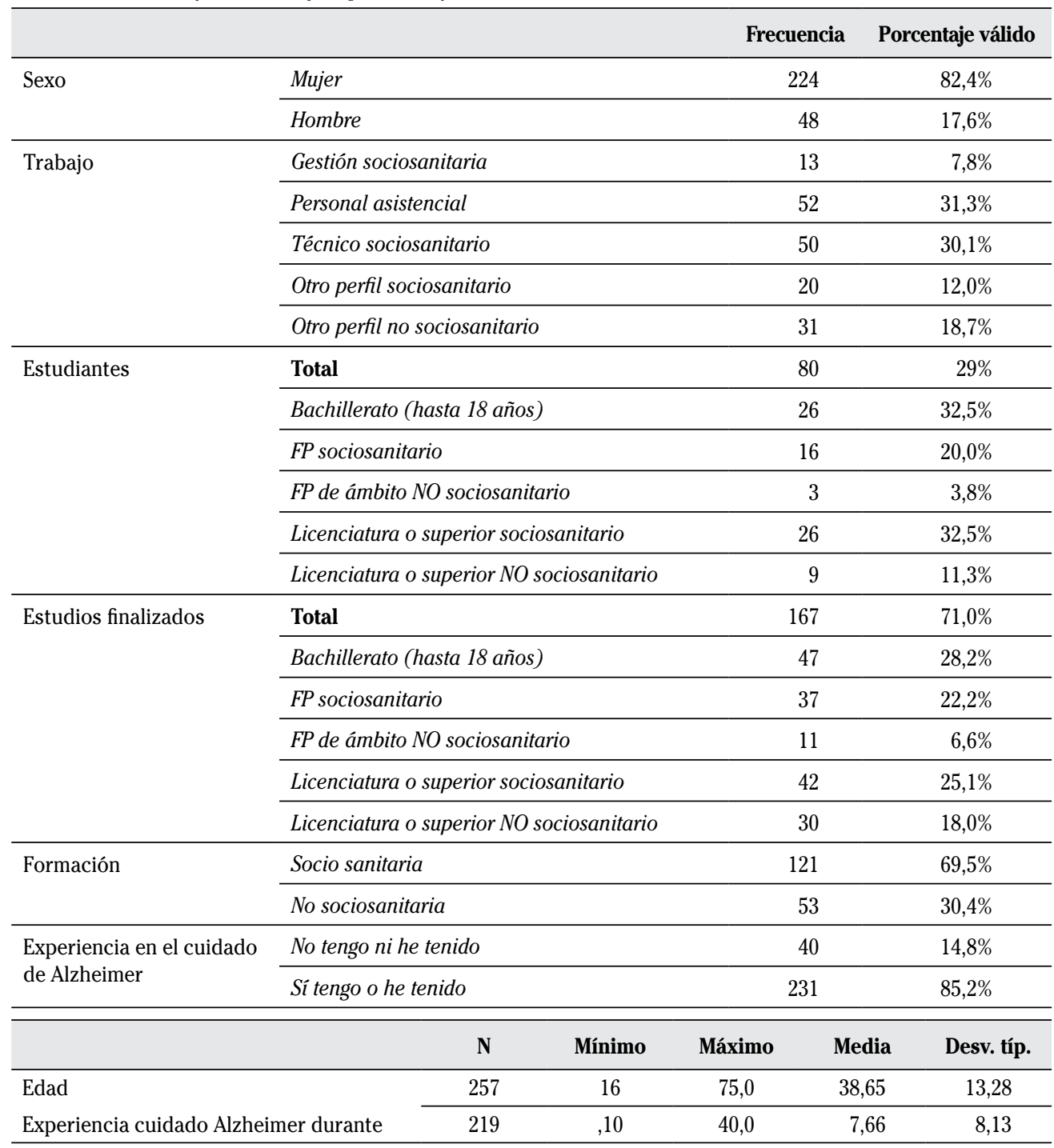

\section{Elaboración del cuestionario}

Tras revisión bibliográfica se redactaron los ítems contando con la colaboración y experiencia de dos enfermeras de un centro sociosanitario con 130 camas de uso geriátrico. Después de generar un retrato robot sobre la persona tipo a favor y en contra del uso de las sujeciones, se redactaron distintos ítems que hacían referencia a las posibles conductas, emociones y cogniciones de ambos tipos. Durante el proceso de validación de contenido (o exploración de si el instrumento mide el concepto que quiere medir $^{20}$ ) se envió el listado de ítems a distintos jueces expertos en uso y restricción de sujeciones, para que pudieran revisar, corregir y/o aportar información. Éstos analizaron los ítems redactados como opciones válidas o no para las distintas explicaciones 
que los profesionales y/o trabajadores dan al mismo. Se realizó en dos fases. En la primera eliminaron, añadieron y modificaron ítems. En la segunda se les envió el ejercicio definitivo para que mostraran su conformidad con el resultado final.

Para u tilizar metodología equivalente a otros estudios se especificó en las instrucciones escritas previas lo siguiente: "Tenga en cuenta que por SUJECIÓN entendemos restricciones físicas y/o farmacológicas (excepto barandillas)".

El cuestionario de 49 ítems se puntuaba del 1 al 5 siendo 1 Totalmente en desacuerdo y 5 Totalmente de acuerdo. Los ítems podían ser directos o inversos de tal forma que obtener mayores puntuaciones en la escala se correspondía con tener una actitud a favor de la liberación de las sujeciones o, lo que es igual, en contra de su uso. Se añadieron otras preguntas sobre variables sociodemográficas (edad y sexo), formación, experiencia laboral y en el cuidado del Alzheimer para conocer el perfil exacto de la muestra.

Además, con el objetivo de obtener evidencias sobre validez en relación con otras variables se añadieron dos preguntas: "En su planta, ¿cuántos pacientes usan sujeción?” y "¿cuántos cree que realmente necesitan sujeción?". Si la variable "Diferencia", resultante de restar los dos valores obtenidos ofreciera un valor positivo indicaría actitud en contra del uso de las sujeciones (ya que hay más personas en mi planta con sujeciones de las que debería haber). Por el contrario, un valor negativo indicaría actitud a favor de su uso, lo mismo (aunque más discutible) que un valor de cero, ya que en este caso también se está de acuerdo con el uso de hecho que se hace de la sujeción.

El cuestionario se repartió a todos los asistentes a las jornadas junto con la documentación entregada en las mesas de inscripción. Se pidió la colaboración y que lo entregaran al finalizar las mismas.

\section{Análisis estadísticos}

Se utilizó el paquete estadístico SPSS v18.0. Para la validación de la escala se realizaron los siguientes análisis. Se analizó la fiabilidad tanto de la escala como de las subescalas mediante análisis de consistencia interna (coeficiente alfa de Cronbach). Se analizó la validez de la estructura interna mediante análisis factorial exploratorio utilizando el método de componentes principales y rotación varimax. Previo al análisis factorial se realizaron las pruebas de adecuación muestral Kaiser-Meyer-Oklin y de esfericidad de Barthlett. Se asumió un nivel de significación estadístico de $\mathrm{p}<0,05$.

Para obtener evidencias de relaciones con criterios externos, se utilizó la variable "Diferencia", comparando los 3 grupos resultantes mediante ANOVA de un factor y HSD de Tukey (las pruebas Levene permitieron asumir la igualdad de varianzas). Se contrastó así mismo la diferencia entre medias de las variables sexo y experiencia en Alzheimer mediante $t$ de Student para muestras independientes.

\section{RESULTADOS}

Con una muestra final de 276 cuestionarios, la tasa de respuesta fue del $65,5 \%$. La muestra mayoritariamente femenina con $82,4 \%$ (224) de mujeres se distribuyó respecto al perfil laboral de la siguiente manera; de los 166 que contestaron "mi trabajo es”, la mayor parte, 135 (81,3\%) marcaron perfil sociosanitario, mientras que 31 $(18,7 \%)$ seleccionaron otros perfiles. Quince personas del total de la muestra eran voluntarios de la casa $(5,4 \%)$.

Respecto a la formación, 80 (29\%) eran estudiantes, de los cuales 42 (52,5\%) cursaban estudios sociosanitarios y 167 (71\%), marcaron sus estudios como finalizados, $79(47,3 \%)$ de ellos en el ámbito sociosanitario. Por lo tanto en nuestra muestra contamos con una tercera parte sin formación sociosanitaria y el resto sociosanitaria (Tabla 1).

Por último, en nuestra muestra, 231 personas $(85,2 \%)$ manifestaron tener experiencia en el cuidado del Alzheimer con una media de tiempo de 7,6 años (se especificaba que podía ser tanto en el cuidado de un familiar como por experiencia profesional). 
Durante el análisis de fiabilidad se eliminaron 9 ítems que no contribuían a la homogeneidad del test, elevándose el valor del índice alfa de Cronbach a 0,897 en un cuestionario final de 40 ítems (Tabla 2).

Tabla 2. Análisis de fiabilidad de escalas y subescalas, numero de ítems que las componen y medidas descriptivas para cada una de ellas

\begin{tabular}{lccccc}
\hline & $\begin{array}{c}\text { Factor 1 } \\
\text { Autonomía }\end{array}$ & $\begin{array}{c}\text { Factor 2 } \\
\text { Seguridad }\end{array}$ & $\begin{array}{c}\text { Factor 3 } \\
\text { Entorno }\end{array}$ & $\begin{array}{c}\text { Factor 4 } \\
\text { Evitación }\end{array}$ & $\begin{array}{c}\text { Escala } \\
\text { Global }\end{array}$ \\
\hline $\mathrm{N}$ & 207 & 217 & 238 & 237 & 152 \\
\hline $\mathrm{N}$ ㅇ Ítems & 15 & 11 & 7 & 4 & 40 \\
\hline Alfa & 0,88 & 0,82 & 0,72 & 0,47 & 0,90 \\
\hline Media & 51,10 & 36,73 & 20,91 & 10,69 & 129,74 \\
\hline Mediana & 51 & 37 & 21 & 11 & 127,5 \\
\hline Desv. Típica & 10,86 & 7,47 & 5,32 & 2,97 & 20,98 \\
\hline Media del ítem & 3,41 & 3,34 & 2,99 & 2,67 & 3,24 \\
\hline Mín-Máx obtenidos & $22-73$ & $13-55$ & $7-32$ & $4-18$ & $75-176$ \\
\hline Posibilidad respuestas & $15-75$ & $11-55$ & $7-35$ & $4-20$ & $40-200$ \\
\hline
\end{tabular}

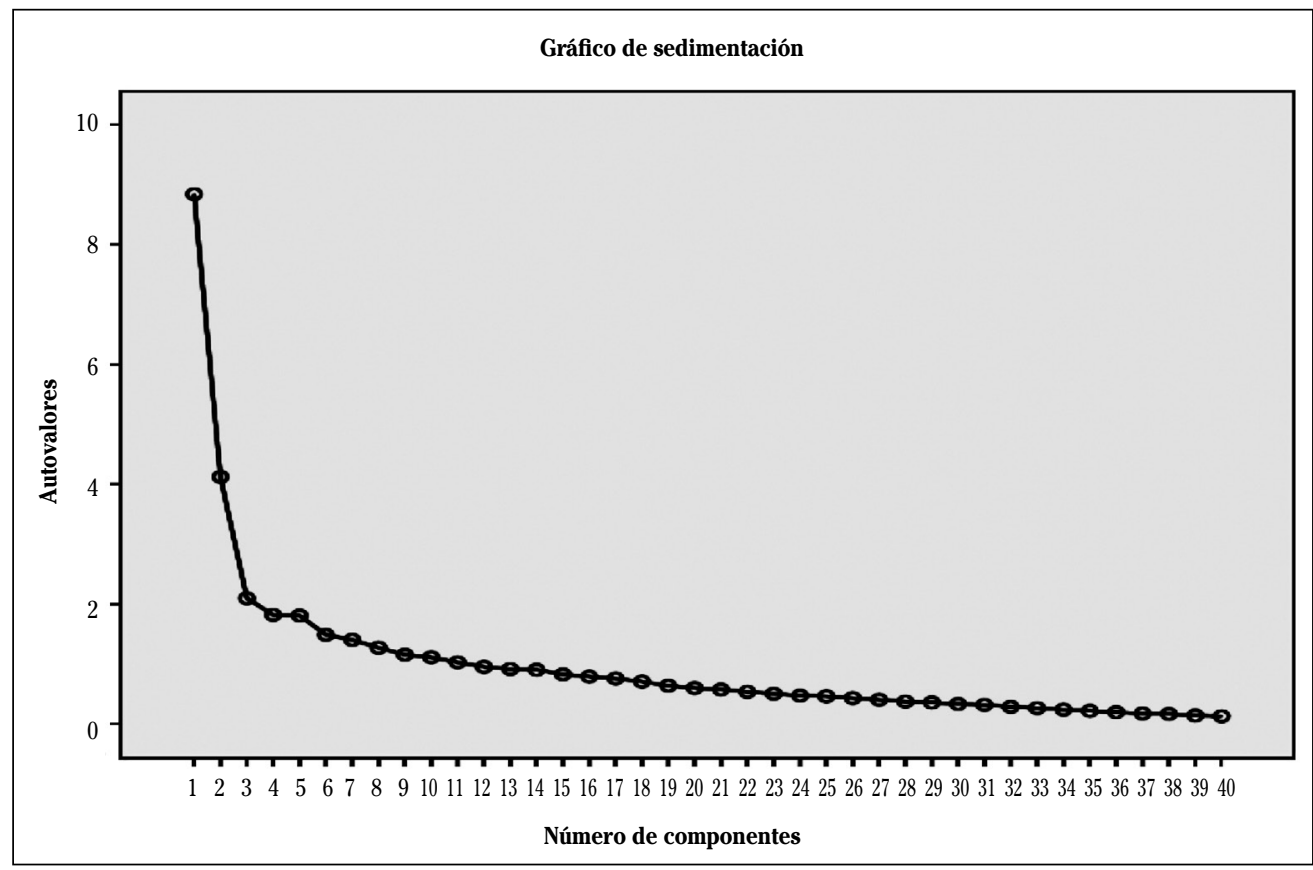

Figura 1. Gráfico de sedimentación de Cattell. 
Ya que de los 276 cuestionarios que constituyeron la muestra final existían valores perdidos al menos en un ítem, aun siendo un criterio estricto, en principio se optó por mantener en el análisis de los datos la supresión de casos según lista, por lo que la muestra válida para el análisis de fiabilidad fue de 152.

La confirmación de la adecuación de los datos quedó garantizada por resultar el valor KMO aceptable $(0,79)$ y la prueba de esfericidad de Barthlett significativa $(0,00)$.

El análisis factorial exploratorio arrojó una escala de 11 factores que explicaba una varianza del $65,48 \%$, mientras que el gráfico de sedimentación dejaba 5 factores a la izquierda del cambio de pendiente. Debido a la mayor coherencia interna de los ítems que conformaban estos 5 factores y mayor parsimonia se optó por la extracción según Cattell (Fig. 1). Las 5 dimensiones (Tabla 3) explicaban un $46,76 \%$ de la varianza total.

El Factor 1, nombrado Autonomía, explica el $15,08 \%$ de la varianza y según la matriz de componentes rotados incluye 15 ítems directos $(8,16,20,21,22,24,25,28$, $29,30,31,33,35,39,40)$ que, entre otras, recogen expresiones de defensa hacia los derechos de la persona dependiente, el derecho de autonomía y las ventajas para el usuario derivadas de evitar utilizar las sujeciones (con un N válido de 207 cuestionarios).
El Factor 2, Seguridad, explica el 12,23\% de la varianza e incluye 11 ítems inver$\operatorname{sos}(5,10,15,17,18,23,32,34,36,37,38$; $\mathrm{N}=217$, que justifican su uso recogiendo la consecuencias negativas para el dependiente si no se usan las sujeciones, y la incomodidad y/o falta de seguridad que siente el profesional al no utilizarlas.

El Factor 3, Entorno, explica el 8,46\% de la varianza e incluye 7 ítems, todos directos menos el 26 que es inverso $(4,7,9,13,19$, 26,$27 ; \mathrm{N}=238$ ) que justifican el uso de las sujeciones debido a la falta de alternativas para el entorno, familiares y profesionales.

El Factor 4, Evitación, explica 6,06\% de la varianza e incluye 4 ítems inversos $(1,6$, 12,$14 ; \mathrm{N}=237$ ) que ponen la justificación de su uso en manos de otros, posiblemente evitando la manifestar la propia actitud, que de fondo se muestra a favor del uso de las sujeciones.

Así, la redacción de los ítems pertenecientes a los factores 1 y 3 están a favor de la liberación (o en contra del uso de las sujeciones), mientras que la de los factores 2 y 4 principalmente defienden su uso. El Factor 5, que explica el 4,92\% de la varianza no se interpretó por no llegar a incluir 3 ítems con una saturación mayor de 0,35.

Los ítems ambiguos guardaron más coherencia con el factor al que en principio fueron asignados por lo que se respetó su ubicación (Tabla 3).

Tabla 3. Factores resultantes del Análisis factorial exploratorio. Matriz de componentes rotados, autovalores y porcentaje de la varianza explicados por cada uno. Los ítems inversos se muestran en cursiva.

\begin{tabular}{|c|c|c|c|c|c|}
\hline & \multicolumn{5}{|c|}{ Factores } \\
\hline & 1 & 2 & 3 & 4 & 5 \\
\hline 30. Si estuviese el personal bien formado y tuviera paciencia no serían tan necesarias & 0,74 & & & & \\
\hline 33. Sin sujeciones mejoraría la satisfacción tanto de los usuarios como de sus familias & 0,71 & & & & \\
\hline 25. No usarlas mejoraría el estado de ánimo del residente & 0,71 & & & & \\
\hline 29. Las sujeciones pueden constituir una violación de los derechos fundamentales & 0,66 & & & & \\
\hline 28. Provoca en el paciente efectos adversos físicos, psicoemocionales y sociales & 0,66 & & & & \\
\hline $\begin{array}{l}\text { 16. Las sujeciones son algo que vulnera un derecho fundamental, como es el derecho } \\
\text { a la libertad }\end{array}$ & 0,63 & & & & \\
\hline 22. Me gusta el movimiento "desatar a la persona mayor" & 0,59 & & & & \\
\hline 31. Si se eliminaran las prácticas restrictivas mejorarían el equilibrio & 0,59 & & 0,36 & & \\
\hline
\end{tabular}




\begin{tabular}{|c|c|c|c|c|c|}
\hline & \multicolumn{5}{|c|}{ Factores } \\
\hline & 1 & 2 & 3 & 4 & 5 \\
\hline 21. Me asusta la desmotivación del personal que lleva a mayor uso de sujeciones & 0,57 & & & & \\
\hline 20. Los residentes estarían mejor sin sujeción por su propio bien & 0,50 & & & & \\
\hline 8. La ausencia de normas al respecto hace que se pongan más sujeciones de lo debido & 0,50 & & & & \\
\hline 40. En un centro libre de sujeciones hay mejor ambiente entre los trabajadores & 0,47 & & & & \\
\hline 24. No existe ninguna prueba de que las sujeciones sean efectivas & 0,46 & & 0,37 & & \\
\hline 35. Un buen paseo es mejor que un chaleco & 0,43 & 0,35 & & & $-0,40$ \\
\hline 39. Aceptaría actividades formativas encaminadas a la reducción de las sujeciones & 0,34 & & & & \\
\hline 18. Los pacientes estân más tranquilos si están medicados y sujetos & & 0,72 & & & \\
\hline 37. Las sujeciones neutralizan conflictos de convivencia & & 0,68 & & & \\
\hline $\begin{array}{l}\text { 23. Me siento más cómodo si están los pacientes sujetos ya que evita agresiones entre } \\
\text { ellos }\end{array}$ & & 0,65 & & & \\
\hline 32. Siento cierta incomodidad o cierto miedo al retirar una sujeción & & 0,62 & & & \\
\hline $\begin{array}{l}\text { 36. La utilización de sujeciones mejora el control de las personas mayores o con } \\
\text { discapacidad }\end{array}$ & & 0,61 & & & \\
\hline 17. Los centros que utilizan sujeciones son menos vulnerables a las sanciones legales & & 0,60 & & & \\
\hline 10. Las sujeciones corrigen la postura & & 0,57 & & & \\
\hline 15. Las sujeciones protegen a la persona dependiente & & 0,56 & & & \\
\hline 38. Los profesionales deberíamos tener más autonomía para poner sujeciones & & 0,53 & & & \\
\hline 34. Suelo demandar medicación para algunos pacientes & & 0,53 & & & \\
\hline 5. El uso de sujeciones me da seguridad en el día a día del trabajo & & 0,48 & & 0,37 & 0,37 \\
\hline 13. Las sujeciones obedecen a la autoprotección de los profesionales & & & 0,65 & & \\
\hline $\begin{array}{l}\text { 9. Las familias acaban aceptando las sujeciones porque no se les ofrecen otras } \\
\text { alternativas }\end{array}$ & & & 0,65 & & \\
\hline 7. Habría que tener en cuenta la opinión del residente & & & 0,6 & & \\
\hline 26. Normalmente está justificado el uso de sujeciones & & & 0,59 & & \\
\hline 27. Las sujeciones provocan efectos negativos en los profesionales de la salud & & & 0,56 & & \\
\hline 19. Los profesionales no toleramos la inquietud de los residentes & 0,39 & & 0,45 & & \\
\hline $\begin{array}{l}\text { 4. El uso de sujeciones aumenta la fragilidad del paciente, aumentando el riesgo de } \\
\text { caídas }\end{array}$ & & & 0,39 & & \\
\hline 6. Existe reticencia a pautar medicación para contener al paciente & & & & 0,75 & \\
\hline 1. A veces tengo que dar muchas justificaciones para que prescriban una sujeción & & & & 0,55 & \\
\hline 14. Las sujeciones previenen la deambulación con riesgo & & & & 0,41 & \\
\hline 12. Las familias prefieren el uso de sujeciones para evitar caídas, fugas, etc. & & & & 0,37 & \\
\hline 3. Atender a las personas es más eficiente mediante el uso de sujeciones & & & & & 0,76 \\
\hline 2. Antes de pautar sujeción hay que buscar alternativas & & & & $-0,38$ & 0,43 \\
\hline $\begin{array}{l}\text { 11. Las sujeciones crean mayor dependencia en el paciente, necesitando éste mayor } \\
\text { cuidado }\end{array}$ & & & & & 0,31 \\
\hline Autovalor & 6,03 & 4,89 & 3,38 & 2,42 & 1,96 \\
\hline Porcentaje de la varianza explicado & 15,08 & 12,22 & 8,46 & 6,06 & 4,92 \\
\hline
\end{tabular}

1: Autonomía. 2: Seguridad. 3: Entorno 4: Evitación. 5: Global. 
El cuestionario final por tanto recoge dos factores a favor y dos en contra de la liberación de las sujeciones pero, una vez invertidas las puntuaciones de los 17 ítems inversos del cuestionario (Tabla 3, en cursiva), valores altos en cualquiera de los factores como en la escala global indican actitud favorable hacia la liberación de las sujeciones.

La media obtenida para el total de la escala es de 129,74 puntos (mediana 127,5 y DT 20,98), con un mínimo de 75 y un máximo de 176 habiendo posibilidad de respuestas entre 40 y 200 puntos. La media de puntuación de los ítems es de 3,24 (Tabla 2).

La distribución de puntuaciones, las posibilidades de respuesta, el máximo y mínimo que se pueden encontrar en cada factor y la media de puntuación de los ítems por factor se muestran en la misma tabla (del primer factor: 3,41 ; segundo: 3,34 ; tercero: 2,99 y cuarto: 2,67 ).

Existen correlaciones de Pearson significativas (nivel 0,001 bilateral) entre escala y subescalas. De la puntuación total con el factor $1(\mathrm{P}=0,85)$ muy elevada y directa. Aceptable con el factor $2(\mathrm{P}=0,68)$ y $3(\mathrm{P}=0,70)$ y moderada con el factor $4(\mathrm{P}=0,48)$.

Respecto a la variable "Diferencia", solamente el $32,6 \%$ de la muestra (90 perso- nas), rellenó estos campos y otro tercio de la muestra (99 personas) confirmó que no trabaja en planta (por lo que no pudo contestar).

Aun así, se obtuvieron resultados estadísticamente significativos. Se crearon tres grupos en la variable de la "Diferencia"; 30 personas estaban en contra del uso de las sujeciones y 12 a favor. El resto, 48 personas, creían que en su planta se utiliza el número adecuado de sujeciones (Tabla 4).

Por un lado, la distribución de puntuaciones totales de los tres grupos se muestra en los diagramas de caja (Fig. 2). Por el otro (una vez asumida la igualdad de varianzas para escala y subescalas mediante la prueba de Levene), se realizó ANOVA de un factor, que mostró la existencia de diferencias significativas entre las puntuaciones de los tres grupos para todas las escalas a excepción del factor 4, evitación. Las pruebas "post hoc", seleccionando HSD Tukey para las comparaciones múltiples, ofrecieron los resultados que se muestran en la tabla 4. El primer grupo (supuestamente a favor de la liberación, puntuó significativamente $(\mathrm{p}<0,05)$ más alto que segundo (en principio conforme) y tercero (teóricamente en contra de la liberación), en todas las escalas (a excepción de la cuarta, evitación).

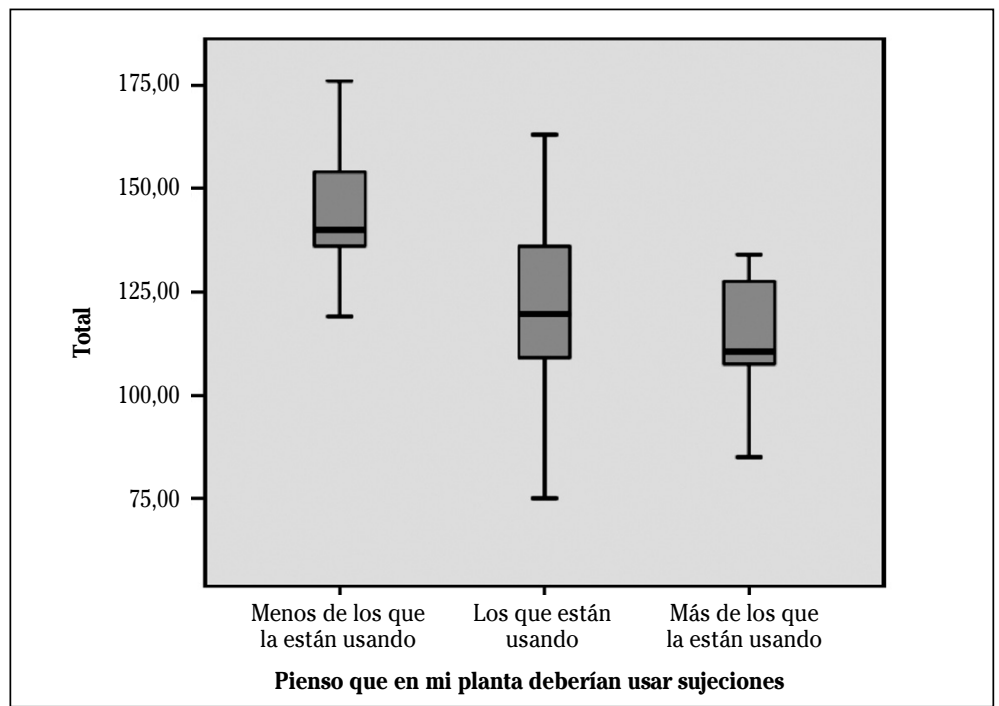

Figura 2. Diagramas de caja. Distribución de las puntuaciones totales de los 3 grupos que se obtienen en la variable Diferencia, en función de la opinión respecto al uso de sujeciones en la planta de trabajo. 
En esta misma tabla se observa que no existen diferencias significativas por razones de edad, ni por años de experiencia, ni debido a la formación. Sí que existen diferencias entre personal con y sin experiencia en el cuidado del Alzheimer. El personal con experiencia puntúa diferencialmente más bajo en factor 1 y factor 3 y más alto en factor 2, pero no hay diferencias en el cómputo global. También existen diferencias entre hombres y mujeres en cuanto al factor 1, donde las mujeres puntúan significativamente más alto (Tabla 4).

Tabla 4. a) Resultados HSD Tukey para escala y subescalas entre los 3 grupos que se generan en función de la opinión respecto al uso de sujeciones en la planta de trabajo y contraste de medias t de Student para muestras independientes para escala y subescalas entre los grupos que se generan en función de b) la experiencia en el cuidado del Alzheimer y c) el sexo

\begin{tabular}{|c|c|c|c|c|c|c|c|}
\hline & \multicolumn{3}{|c|}{$\begin{array}{l}\text { a) Pienso que en mi planta } \\
\text { deberían usar sujeciones }\end{array}$} & \multicolumn{2}{|c|}{$\begin{array}{l}\text { b) Experiencia } \\
\text { en el cuidado de } \\
\text { Alzheimer }\end{array}$} & \multicolumn{2}{|c|}{ c) Sexo } \\
\hline & $\begin{array}{c}\text { Menos de } \\
\text { los que la } \\
\text { están usando } \\
(\mathrm{N}=30)\end{array}$ & $\begin{array}{l}\text { Los que la } \\
\text { están usando } \\
(\mathrm{N}=48)\end{array}$ & $\begin{array}{c}\text { Más de los } \\
\text { que la están } \\
\text { usando } \\
(\mathrm{N}=12)\end{array}$ & $\begin{array}{c}\text { No } \\
(\mathrm{N}=40)\end{array}$ & $\begin{array}{c}\text { Sí } \\
(\mathrm{N}=231)\end{array}$ & $\begin{array}{l}\text { Mujer } \\
(\mathrm{N}=224)\end{array}$ & $\begin{array}{l}\text { Hombre } \\
(\mathrm{N}=48)\end{array}$ \\
\hline & Media & Media & Media & Media & Media & Media & Media \\
\hline $\begin{array}{l}\text { Factor } 1 \\
\text { Autonomía }\end{array}$ & $54,85^{\mathrm{a}}$ & $45,29^{b}$ & $41,50^{b}$ & $54,67^{\mathrm{a}}$ & $50,47^{\mathrm{a}}$ & $52,03^{a}$ & $48,12^{b}$ \\
\hline $\begin{array}{l}\text { Factor } 2 \\
\text { Seguridad }\end{array}$ & $39,85^{\mathrm{a}}$ & $34,91^{b}$ & $34,78^{\mathrm{a}, \mathrm{b}}$ & $33,10^{a}$ & $37,3^{b}$ & $36,73^{\mathrm{a}}$ & $36,52^{\mathrm{a}}$ \\
\hline $\begin{array}{l}\text { Factor } 3 \\
\text { Entorno } \\
\end{array}$ & $22,89^{\mathrm{a}}$ & $18,18^{b}$ & $17, \mathbf{3 3}^{\mathbf{b}}$ & $22,57^{a}$ & $20,64^{b}$ & $20,96^{\mathrm{a}}$ & $20,89^{\mathrm{a}}$ \\
\hline $\begin{array}{l}\text { Factor } 4 \\
\text { Evitación }\end{array}$ & $10,96^{\mathrm{a}}$ & $9,76^{\mathrm{a}}$ & $10,10^{\mathrm{a}}$ & $10,45^{\mathrm{a}}$ & $10,68^{\mathrm{a}}$ & $10,67^{\mathrm{a}}$ & $10,63^{\mathrm{a}}$ \\
\hline $\begin{array}{l}\text { Escala } \\
\text { Global }\end{array}$ & $142,81^{a}$ & $119,65^{b}$ & $113,75^{b}$ & $130,19^{\mathrm{a}}$ & $129,56^{\mathrm{a}}$ & $131,06^{\mathrm{a}}$ & $124,77^{\mathrm{a}}$ \\
\hline
\end{tabular}

Nota: Los valores de la misma fila y subtabla que no comparten el mismo superíndice (y en negrita) son significativamente diferentes en $\mathrm{p}<0.05$ en la prueba de igualdad bilateral de medias de las columnas. Las pruebas asumen varianzas iguales.

\section{DISCUSIÓN}

En este estudio se ha diseñado y validado una herramienta de medida de la actitud del personal asistencial hacia el uso de sujeciones físicas o farmacológicas. Se ha obtenido una escala fiable y con una estructura interna de 4 factores que explican de manera coherente y parsimoniosa las actitudes básicas que se recogen en la literatura ${ }^{16}$ : la defensa de la autonomía y los derechos de los pacientes (factor 1), en el otro lado la seguridad que el profesional necesita tener para no verse desbordado en su trabajo en el día a día (factor 2), las influencias o presiones que recibe el pro- fesional por parte del entorno garantista o protector con el que convive (factor 3 ) y la posible evitación a la que necesita recurrir también en ocasiones para eludir la carga emocional y/o burnout (factor 4).

Sobre la adecuación de la muestra es importante resaltar que solamente el $15 \%$ (40 personas) no ha tenido ni tiene experiencia en el cuidado del Alzheimer, de los cuales solamente 7 (2,7\%) personas no tienen ni formación sociosanitaria ni trabajo con perfil sociosanitario, por lo que constituyendo una muestra heterogénea, prácticamente la totalidad está vinculada con el cuidado de personas dependientes (ge- 
neralmente Alzheimer) y conocen o experimentan el conflicto en el uso de sujeciones.

El análisis factorial es adecuado y el modelo consigue un buen ajuste por lo que entendemos que existen evidencias respecto a la validez de estructura interna del cuestionario. En cualquier caso, convendría ampliar ítems en el factor 4, cuya consistencia interna no es muy elevada debido a su longitud (solamente integra 4 ítems) y su comportamiento no queda claramente explicado. Por un lado en los mismos contrastes de medias donde el resto de escalas muestran diferencias discriminando una actitud a favor o en contra del uso de sujeciones, el factor 4 no muestra diferencias y por otro lado es la única subescala que correlaciona por debajo de un nivel aceptable $(\mathrm{P}<0,65)$ con la puntuación total del cuestionario.

La validez formulada en términos de relaciones con otras variables, queda contrastada a lo largo de los análisis realizados, que esperan y obtienen diferencias significativas entre las medias de las puntuaciones de los grupos según el juicio que hacen hacia el uso de sujeciones en sus plantas. El grupo que responde. Pienso que en mi planta deberían usar sujeciones... menos de los que la están usando, que parece que en su práctica cotidiana se muestra a favor de la liberación, puntúa significativamente más alto en todas las escalas (a excepción de la cuarta), que los otros dos grupos, el que responde Pienso que en mi planta deberían usar sujeciones... los mismos que la están usando, al que podríamos adjetivar como conformista, y... más de los que la están usando, del que podríamos decir, tiene actitud contraria a la liberación de las sujeciones en su práctica cotidiana.

Además, lo que se contrasta mediante el ANOVA de un factor, se confirma visualmente mediante los diagramas de cajas, que permiten observar las diferencias y semejanzas en las distribuciones de las puntuaciones que se obtienen en cada grupo.

El hecho de que esta parte de la muestra que trabaja en planta (y por tanto se somete a los análisis del estudio) sea escasa, al menos en uno de los grupos creados, constituye, junto con la longitud del factor cuarto, una limitación en el estudio.

Aun así la validez de la escala se sostiene también en la distribución de los ítems en la rotación, que agrupa precisamente las dimensiones a los que la literatura se refiere como de mayor conflicto y con pesos equitativos, por un lado la defensa de la autonomía y los derechos de los pacientes con un $15 \%$ y por otro la protección de la persona que está al cuidado con un $12 \%$ de la explicación de las variaciones en las respuestas.

Validez corroborada por los resultados en los contrastes de medias entre los que tienen experiencia en el cuidado del Alzheimer (con una media de 7,6 años de cuidados) que puntúan más alto en seguridad y más bajo en autonomía que los que no tienen dicha experiencia (o no se han visto comprometidos en el día a día laboral por los distintos riesgos de la liberación). Aun así este último resultado habría de interpretarse con cautela ya que las medias que se comparan tienen un $\mathrm{N}$ válido claramente descompensado (231 vs 40).

La escala generada, a medida que vaya acumulando evidencias sobre su validez, podría constituir una forma de acercamiento a los pacientes en la atención sanitaria ${ }^{24,25}$, a través tanto de la detección de posibles necesidades de formación, de generación de cambios en las actitudes existentes, o para su uso en la investigación ${ }^{26-29}$.

En resumen, no exenta de limitaciones, esta escala constituye un paso significativo en el estudio de las actitudes de los profesionales asistenciales hacia el uso de sujeciones físicas y farmacológicas, indicando no solo cuál es la actitud hacia la liberación de las sujeciones sino profundizando también en las causas subyacentes ya que diferencia si existe falta de convicción sobre los derechos de la persona dependiente, exceso de celo en la seguridad del dependiente o presiones asociadas sobretodo al entorno.

\section{BIBLIOGRAFÍA}

1. HAMERS JP, GULPERS MJ, y StRIK, W. Use of physical restraints with cognitively impaired nursing homes residents. J Adv Nurs 2004; 45: 246-251. 
2. Engberg J, Castle N, McCaffrey D. Physical restraint initiation in nursing homes and sub sequent resident health. Gerontologist 2008; 48 : 442-452.

3. Burgueño AA. Memoria del Programa Desatar al Anciano y al Enfermo de Alzheimer. Confederación Española de Organizaciones de Mayores 2007. [consultado 05-06-2013]: Disponible en: http://www.ceoma.org/alzheimer/index.php

4. Galán CM, Trinidad D, Ramos P, Gómez JP, AlasTRUEY J, OnRUBiaA ET AL. Uso de sujeciones físicas en una población anciana ingresada en residencias públicas. Rev Esp Geriatr Gerontol 2008; 43: 208-213.

5. Vidán MT, SÁnchez E, Alonso M, Montero B, MartíNEZ dE la CASA A, ORTiz FJ ET AL. Deterioro funcional durante la hospitalización en ancianos: beneficios del ingreso en el servicio de geriatría. Rev Esp Geriatr Gerontol 2008; 43: 133-138.

6. TinetTI ME, Liu WL, GinTER SF. Mechanical restraint use and fall-related injuries among residents of skilled nursing facilities. Ann Intern Med 1992; 116: 369-374.

7. Capezuti E, Strumpf NE, Evans LK, Grisso JA, MAISLIN G. The relation ship between physical restraint removal and falls and injuries among nursing home residents. J Gerontol A Biol Sci Med Sci 1998; 53: M47-52.

8. Capezuti E, Evans L, Strumpf N. Physical restraint use and falls in nursing home residents. J Am Geriatr Soc 1996; 44: 627-633.

9. COTTER VT. Restraint free care in older adults with dementia. Keio J Med 2005; 54: 80-84.

10. Knox, J. Reducing physical restraint use in residential aged care: implementation of an evidence-based approach to improve practice. Int J Evid Based Healthc 2007; 5: 102-107.

11. Kосн S, Lyon C, Lyon K.S. Case study approach to removing physical restraint. Int J Nurs Pract 2001; 7: 156-161. http://www.scopus.com.scopeesprx.elsevier.com/search/submit/citedby. url?eid=2-s2.0-84872350129\&refeid=2-s2.0$0035383379 \&$ src $=$ s\&origin $=$ reflist $\&$ refstat $=$ core

12. Косн S, NAY R, WiLSON, J. Restraint removal: tension between protective custody and human rights. Int J Older People Nurs 2006; 1: 151-158.

13. Burgueño AA, Iborra I, Martínez P, Pérez V. Prevalencia comunicada de sujeciones físicas: Resultado de la aplicación de un sistema de información para un benchmarking anónimo entre residencias españolas del Programa desatar al anciano y al enfermo de Alzheimer. Agathos: Atención sociosanitaria y bienestar 2008; 1: 4-11.

14. The University of Texas MD Anderson Cancer Center Restraints Improvement Group: Alternatives for Physical Restraint: Myths and Truths about Physical Restraints; Including a
Nursing Survey on Restraint Practices. ISPUB 1999; 3 .

15. Karlsson S, Bucht G, Eriksson S, Sandman P. Factors relating to the use of physical restraints in geriatric care settings. J Am Geriatr Soc 2001; 49: 1722-1728.

16. Haut A, Kolbe N, Strupeit S, Mayer H, Meyer G. Attitudes of relatives of nursing home residents toward physical restraints. J Nurs Scholarsh 2010; 42: 448-456.

17. Chuang Y, Huang H. Nurses' feelings and thoughts about using physical restraints on hospitalized older patients. J Clin Nurs 2007; 16: 486-494.

18. Prat F. Bioética en residencias. Problemas éticos en la persona mayor. Santander: Sal Terrae, 2008.

19. Bermejo JC, Villacieros M, Carabias R. Experimento de aprendizaje sobre cuestiones éticas a partir de una mesa de diálogo interactivo. Gerokomos 2013; 24 (3): (En prensa)

20. Carvajal A, Centeno C, Watson R, Martínez M, SAnz A. ¿Cómo validar un instrumento de medida de la salud? An Sist Sanit Navar 2011; 34: 63-72.

21. Colton D, Xiong H. Reducing seclusion and restraint: Questionnaire for organizational assessment. J Psychiatr Pract 2010; 16: 358-366.

22. HAMERS J, GulPERS M. Reducing physical restraints in nursing homes. Results of a pilot study. Abstract, 19th IAGG World Congress of Gerontology and Geriatrics. J Nutr Health Aging $2009 ; 13, \mathrm{~S} 17$.

23. Lindenmann R. Freiheitsbeschr “ankende Maßnahmen: Einstellung von Pflegepersonen und Pr“ avalenz in den "offentlichen geriatrischen Institutionen der Stadt Luzern. [Physical restraints: Attitudes of nursing staff and prevalence in state-run geriatric institutions in thecity of Luzern]. Master'sthesis. Maastricht University, Maastricht, the Netherlands 2006.

24. Jovell A. El itinerario de la incertidumbre. An Sist Sanit Navar 2013; 36: 5-7.

25. BERMEJO JC. Cuidar a las personas mayores dependientes. Santander: Sal Terrae. 2002.

26. BAzo MT. Negligencia y malos tratos a las personas mayores en España. Rev Esp Geriatr Gerontol 2001; 36: 8-14.

27. NAPIERKOWSKI D. Restringir la utilización de sujeciones. Nursing 2003; 21: 18-22.

28. GALISKY D. Educación médica en geriatría. Rev Esp Geriatr Geronto 11999; 23: 109-112.

29. Woolhead G, Calnan M, Dieppe P, Tado W. Dignity in older age: what do older people in the United Kingdom think? Age Ageing 2004; 33: 165170 . 